\title{
Mechanical analysis of the joint between Wendelstein 7-X target elements and the Divertor frame structure
}

\author{
M.Smirnow ${ }^{\mathrm{a}}$, M.Kuchelmeister ${ }^{\mathrm{a}}$, J.Boscary ${ }^{\mathrm{a}}$, H.Tittes ${ }^{\mathrm{a}}$, A.Peacock ${ }^{\mathrm{a}}$ \\ ${ }^{a}$ Max Planck Institute for Plasma Physics, EURATOM Association, Boltzmannstr. 2, 85748 Garching, Germany
}

\begin{abstract}
The target elements of the actively cooled high heat flux (HHF) divertor of Wendelstein 7-X are made of CFC (carbon fibre-reinforced carbon composite) tiles bonded to a $\mathrm{CuCrZr}$ heat sink and are mounted onto a support frame. During operation, the power loading will result in the thermal expansion of the target elements. Their attachment to the support frame needs to provide, on the one hand, enough flexibility to allow some movement to release the induced thermal stresses and, on the other hand, to provide enough stiffness to avoid a misalignment of one target element relative to the others. This flexibility is realized by a spring element made of a stack of disc springs together with a sliding support at one of the two or three mounting points. Detailed finite element calculations have shown that the deformation of the heat sink leads to some non-axial deformation of the spring elements. A mechanical test was performed to validate the attachment design under cyclic loading and to measure the deformations typical of the expected deformation of the elements. The outcome of this study is the validation of the design selected for the attachment of the target elements, which survived experimentally the applied mechanical cycling which simulates the thermal cycling under operation.
\end{abstract}

Keywords: Wendelstein 7-X, Stellarator, Divertor, finite element calculation

\section{Introduction}

For long pulse operation of the Wendelstein 7-X (W7$\mathrm{X})$ stellarator it is foreseen to install an actively water cooled high heat flux (HHF) divertor, consisting of individual target modules [1]. Each module is a set of target elements made of CFC tiles bonded to a $\mathrm{CuCrZr}$ heat sink, which are mounted onto a support frame. The divertor is made of 10 similar and discrete units aligned along the field lines. Each unit consists of four main areas, three HHF areas; the vertical target, the horizontal target and the high iota tail together with an intermediate section designed for a lower heat flux [2]. The HHF areas thus consist of 50 to $60 \mathrm{~mm}$ wide target elements hydraulically and mechanically connected to each other in modules. The behavior of the attachment system of the target elements under loading has been calculated and experimentally studied prior in the HHF test facility GLADIS [3], the work presented here sets the focus to the details of the attachment system, the disc spring stack that interfaces the target element with the support frame.

Email address: michael.smirnow@ipp.mpg.de (M.Smirnow)

\section{Design of the target element attachment system}

The attachment of the target elements to the support frame has been designed to provide, on the one hand, enough flexibility to allow some movement to release the thermally induced stresses and, on the other hand, to provide enough stiffness to avoid a misalignment of one target element relatively to the others. Fig. 1 shows a $\mathrm{CAD}$ view of the target modules inside the machine with the adjustment frame on the rear side of the target element.

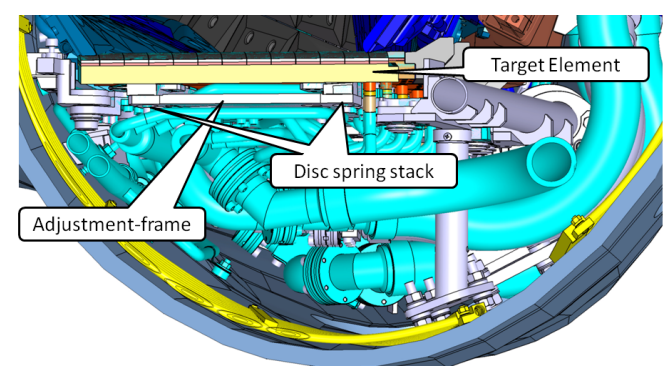

Figure 1: Cross-sectional view through the Divertor at the high iota tail

January 22, 2014 
The target elements have different lengths (from $250 \mathrm{~mm}$ to $600 \mathrm{~mm}$ ) depending on their position in the machine. The number of attachment points depends on the element length: 3 for the long ones $(\leq 500 \mathrm{~mm})$ of the horizontal targets and 2 for the short ones of the high iota tail and of the vertical targets. Finite element calculations which are discussed in detail in section 3.2, show that due to thermal gradients in the target elements during plasma operation, the target bends towards the plasma, causing movements of the element relative to its support frame. As shown in Fig.2, the target elements are manufactured with a $\mathrm{CuCrZr}$ stud and are connected with a M6x18 bolt to the frame via a disc spring stack. This disc spring stack is build up from 6 disc springs (3 serials of 2 parallel pairs) made of Inconel 718 (DIN designation 2.4668). The purpose of this stack is on the one hand to provide flexibility to the thermal induced target movements, and on the other hand to conserve the initial bolt tension.

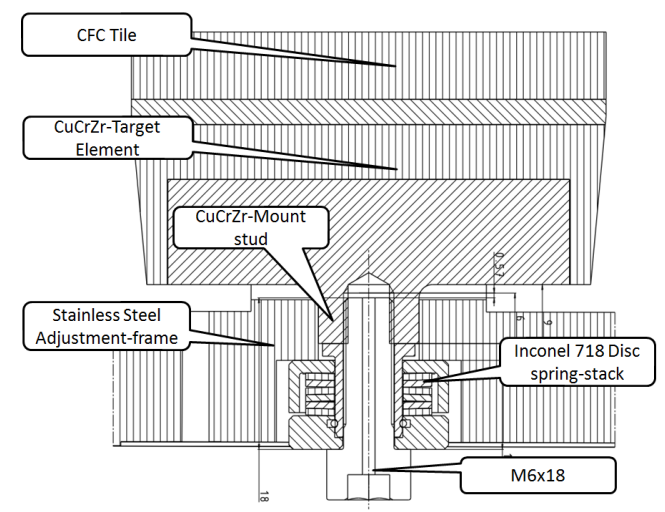

Figure 2: Cross-sectional view through the Target Element and the disc spring stack

The $M 6 x 18$ bolt is tightened for the experiment with a torque wrench to $8 \mathrm{Nm}$ what corresponds to a initial tension of $F_{V}=7 k N$ hence [4]:

$$
F_{V}=\frac{M_{A}}{0,16 P_{h}+0,58 \mu_{g} d_{F}+\mu_{k}\left(\left(d_{a}+d_{i}\right) / 4\right)} .
$$

$M_{A}$ - torque $[\mathrm{Nm}]$

$P_{h}$ - thread pitch [mm]

$\mu_{g}$ - thread friction coefficient $\mu_{g}=0.3$

$\mu_{k}$ - friction coefficient bolt head $\mu_{k}=0.09$

$d_{F}$ - bolt thread diameter [mm]

$d_{a}$ - connecting surface (outer) [mm]

$d_{i}$ - connecting surface (inner) $[\mathrm{mm}]$

The attachment point close to the water connector is fixed, while the other points allow a longitudinal sliding movement to avoid thermal expansion induced stresses.

\section{Simulation of the attachment system response un- der loading}

The mechanical behavior of the target element was analyzed for the specified stationary load of $10 \mathrm{MW} / \mathrm{m}^{2}$ and the $3 \mathrm{~s}$ transient with $16 \mathrm{MW} / \mathrm{m}^{2}$. The type of target element $5 \mathrm{~S}$ (vertical target modules), $55 \mathrm{~mm}$ width and $361 \mathrm{~mm}$ length, armored with $16 \mathrm{CFC}$ tiles, $25 \mathrm{~mm}$ length each, was studied. The heat load was applied on the 3 middle tiles according to the foreseen deposition pattern [5]. Forced convection was used for the inner surface of the cooling channels with a temperature dependent heat transfer coefficient of $\left.\alpha(T)\right|_{60^{\circ} \mathrm{C}}=80 \mathrm{~kW} / \mathrm{m}^{2} \mathrm{~K}$.

The results of the transient calculations show that for the $16 \mathrm{MW} / \mathrm{m}^{2}$ case (worst case scenario) after $3 \mathrm{~s}$ the temperature on the $\mathrm{CFC}$ surface reaches $1250^{\circ} \mathrm{C}, 0.2 \mathrm{~s}$ later the maximum temperature of $350^{\circ} \mathrm{C}$ is reached in the $\mathrm{CuCrZr}$ heat-sink while the rear side of the heat-sink remains at the water temperature. This in-homogeneous temperature distribution causes the heat sink deformation and induces stresses to the attachment points.

\subsection{Modeling of the disc spring stack for ANSYS me- chanical calculation}

Previous calculations, not including the flexibility of the disc springs, had shown that the stress around the studs could exceed $700 \mathrm{MPa}$, leading to possible plastic deformation of the thread [6]. Due to the high geometric non-linearities induced by the non-axial loading of the disc springs inside the stack, it was not possible to bring the calculation to convergence. An alternative approach of using gasket elements was chosen to model the nonlinear behavior of the stack. A tensile testing machine was used to compress the spring stack, while the movement of the machine and the force needed to compress the stack was monitored.

The characteristic curve describing pressure vs. displacement is shown in Fig.3. The step-wise change of the curve after $0.8 \mathrm{~mm}$ is due to the gap between the upper stack cover and the spring bed shown in Fig.2, which is closed after the disc springs become fully compressed.

\subsection{Results of the thermo-mechanical calculation of the element and frame system}

The ANSYS calculation was divided into two load steps. In the first step the bolts were tightened by inserting a force controlled bolt pre-tension boundary condition. The second load step applies the temperature field 


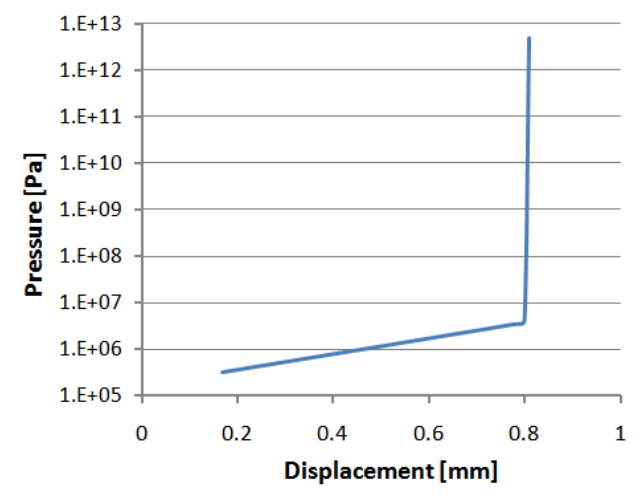

Figure 3: Nonlinear characteristic curve of the disc spring stack modeled as a gasket element in ANSYS

from the thermal analysis, carried out with the material definitions in detail described in [8], at the time 3.2s when the $\mathrm{CuCrZr}$ temperature reaches its maximum. Therefore the contact interface between the bolt and the stud was modeled as a bonded contact, all other contacting bodies as the target element and the disc spring stack to the support frame where treated as rough contacts. Fig.4 shows the temperature field applied as thermal load to the target and the vector-plot of the displacement result-set extracted after the second load step.

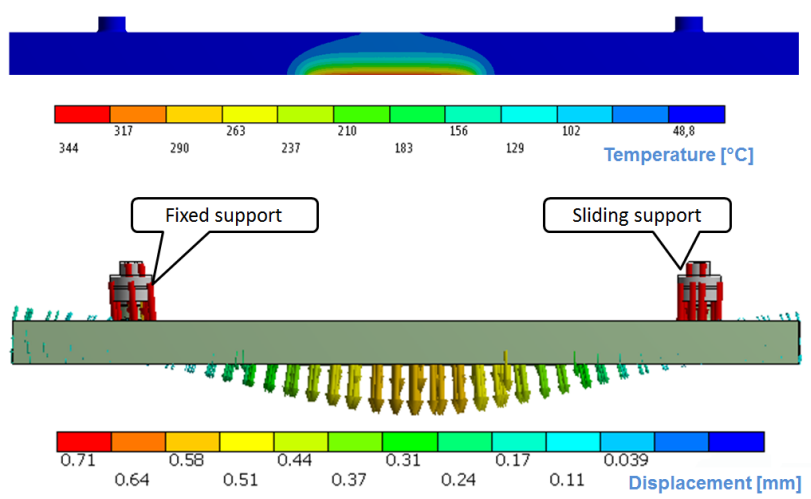

Figure 4: Deformation of the target element due to a $3 \mathrm{~s}$ long $16 \mathrm{MW} / \mathrm{m}^{2}$ pulse load - the elements bends $0.5 \mathrm{~mm}$ towards the plasma

In Fig.4, the fixed stud (water connection side) is on the left. The main displacement is due to the bolt pretension, the non-axial movement of the disc spring stack occurs mainly on the left side. The downward bending of the element is in the range of $0.5 \mathrm{~mm}$. Upward bending occurs on both sides, but is slightly larger on the water connector side. In order to bench mark the calculations it was decided to perform tests to simulate as far as possible the real situation. Fig.5 shows the test device constructed to simulate the cyclic bending of the target element during thermal load. A piston is pressed by the hydraulic actuator of a tensile test machine; the movement and the force are logged by a data acquisition system.

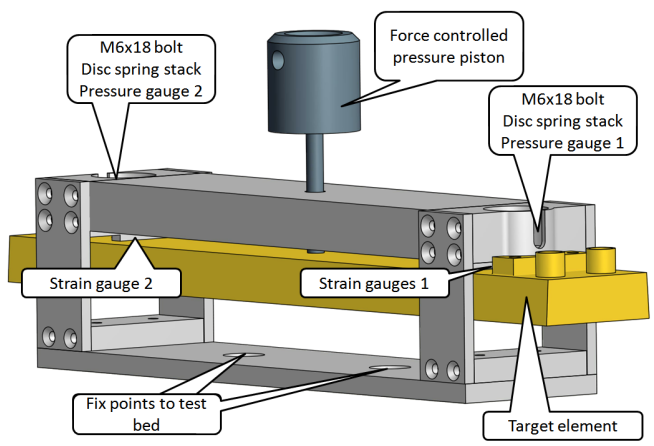

Figure 5: CAD model of the mechanical test device for cyclic load simulation and for the ANSYS simulation. In ANSYS not the piston itself, but the projected area of the pistons contacting surface was used to apply the force directly to the target element

To ensure that this device can simulate the thermal induced deformation of the target element, both cases (stationary and transient loads) were simulated with ANSYS and compared in a design assessment environment. In this environment the deformation vector field (UVECTORS) of the thermal deformation was subtracted from the mechanical deformation vector field [9]. The maximum difference between the two vector fields was $16 \mu m$, which validates the mechanical test device.

Fig. 6 and Fig. 7 show the calculated strain result after the piston moved $0.5 \mathrm{~mm}$ and pushed the target element towards the plasma facing side.

Fig.6 shows strain values in the range of $7.7 \times 10^{-4} \mathrm{~mm} / \mathrm{mm}$, the stud near the water connectors which corresponds to an equivalent stress of $87 M P a$. On the other stud of the element, the force induced strain and stress are $7.5 \times 10^{-5} \mathrm{~mm} / \mathrm{mm}$ and 7.5MPa one magnitude smaller, as shown in Fig.7.

\section{Experimental setup of the bolt test device}

In order to apply a 1-dimensional mechanical load to the target element, a tensile testing machine was modified with a force controlled pressure piston. A test frame was constructed around the target to simulate the same bending as calculated during thermal loading. Fig. 8 shows the test bed with the piston modeled in Fig.5. The simplified model for the ANSYS simulation shown 


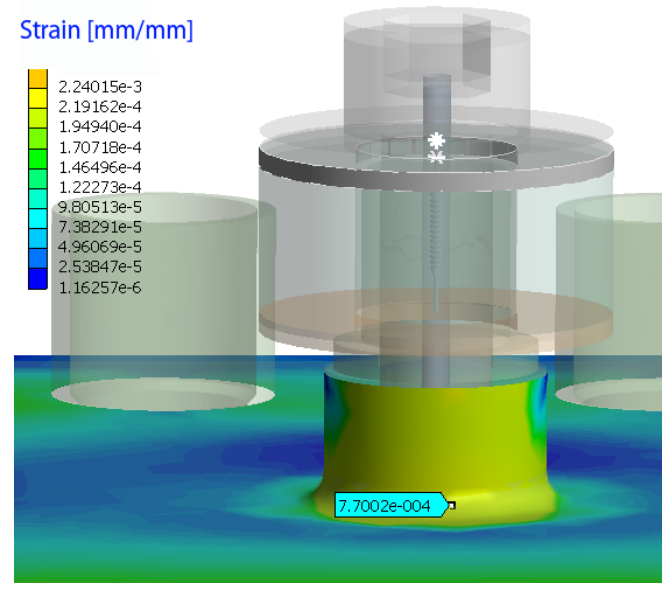

Figure 6: ANSYS strain result of the calculation after load step 2 located on the water connectors side

in Fig.5 excludes the stiffness of the pressure piston itself, because the pressure was applied directly to an area of the pistons size on the surface of the target element. The plugged on attachment of this piston introduced additional degrees of freedom that had to be taken into account. The raw compression vs. vertical movement curve of the piston was measured by pressing it against the tensile test bed's massive machine base. This correction was then added to the overall load configuration shown in the lower part of Fig.9. The load cycle had a trapezoidal shape in time, the piston pressure towards the elements rear side was linear up to $5.5 \mathrm{kN}$, followed by a $20 s$ long flat plateau, then the force decreased linearly back to zero. A second corrective measure has also been applied to the strain gauge measurement values to take into account the gauge temperature dependence given in [7]:

$$
\epsilon_{l}=\frac{a \gamma \alpha \Delta T}{K_{s} R}
$$

$a$ - empiric factor

$\epsilon_{l}$ - strain drift

$\gamma$ - ohmic resistance of the connection

$\alpha$ - temperature coefficient of the connection

$\Delta T$ - temperature difference

$R$ - full resistance of connection plus strain gauge

$K_{s}=\frac{R K}{R-\gamma}-\mathrm{K}$-factor

$K$ - absolute K-factor plus connection

\section{Experimental Results}

A total of 1700 loading and unloading cycles were performed. Fig.9 shows the strain gauge response after

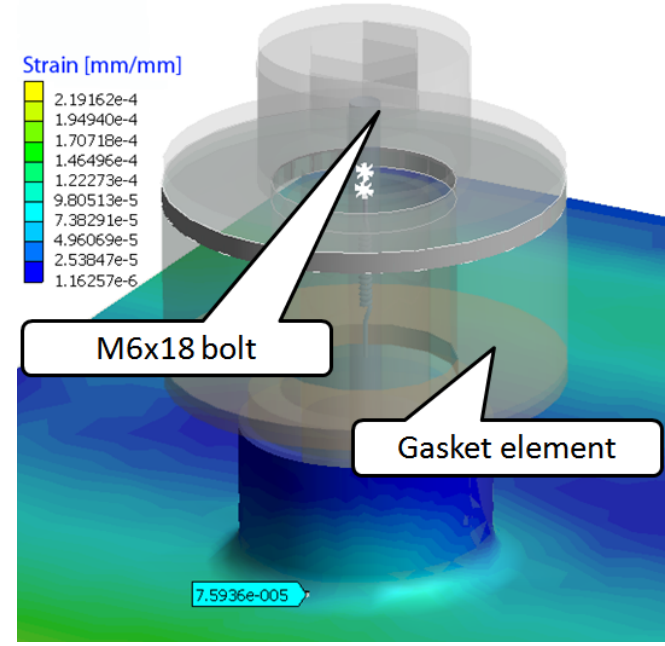

Figure 7: ANSYS strain result of the calculation after load step 2 located on the elements far side

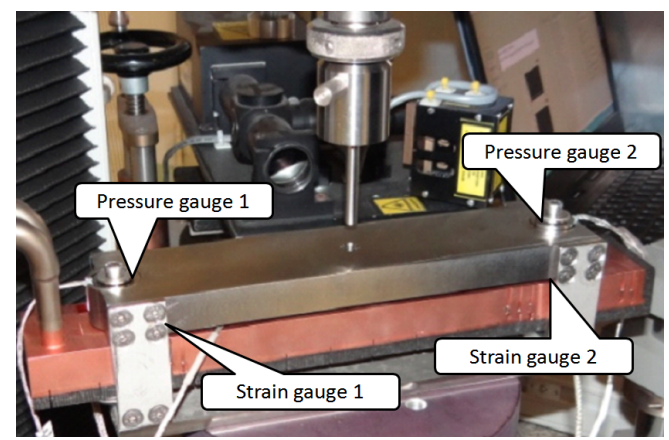

Figure 8: Tensile test bed with sensor equipped target element

250 cycles and Fig. 10 at the end of the test campaign after 1700 cycles. The strain response is very reproducible over 2 consecutive cycles, but shows differences between the strain gauges. As predicted by the ANSYS simulation, the strain measured at the gauge 1 is higher than at the gauge 2 . The gauge 2 shows an overshoot in the ramp up phase before reaching a plateau corresponding to the maximal load value. After 1550 cycles this overshoot of gauge 2 disappeared.

The absolute strain values are comparable with the ANSYS simulation, for the gauge 2, for the gauge 1 the calculation exceeds the measured strain for one magnitude. One reason for this behavior could be the manufacturing tolerance of $+20 \mu m$ of the hole in the support frame, where the target element stud is placed in. This additional tolerance is not included in the CAD model, leading to a higher stiffness of this couple. Visual examination, carried out on the disc springs after the test campaign, showed that there is no material degradation 


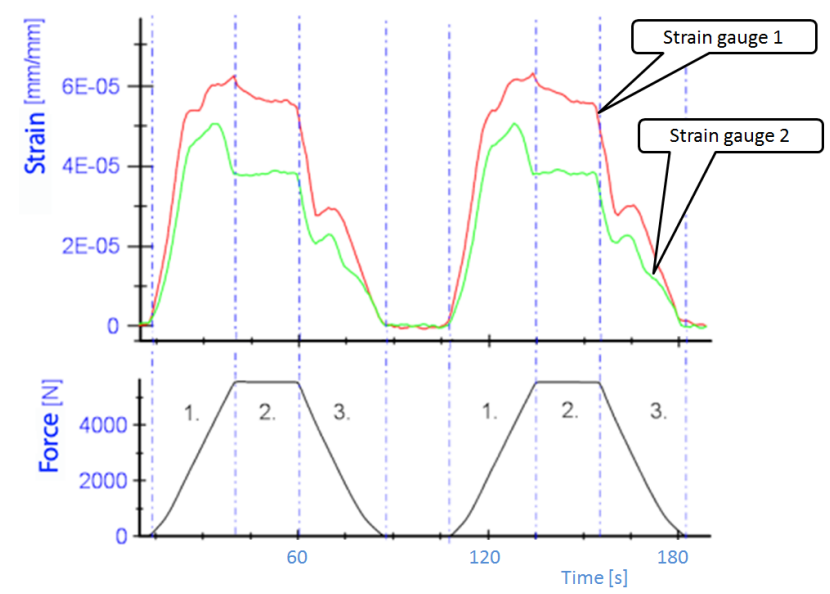

Figure 9: Strain gauge response to load cycle after 250 cycles

visible, their mechanical properties did not change. The measured strain corresponds to stresses in the elastic regime, the target element showed no plastic deformation but a torque measurement during unscrewing of the target element from the support showed a $6 \%$ reduction of the needed torque.

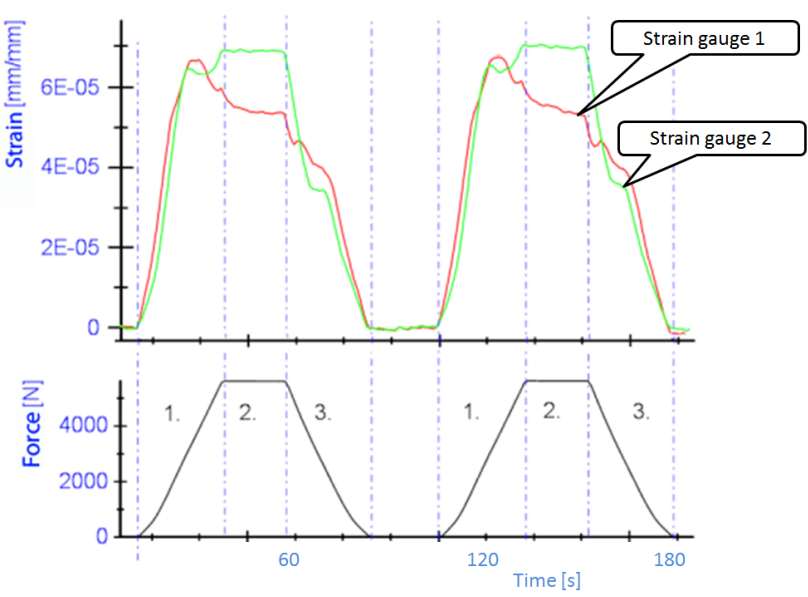

Figure 10: Strain gauge response to load cycle after 1700 cycles

\section{Conclusion}

The behavior of the attachment of the target elements onto the support frame under loading has been modeled and experimentally simulated. The results of this study showed that the designed system fulfills its function and that the attachment maintains the element in its position during thermal cyclic loads. A torque measurement while unscrewing the target element showed that the torque reduced from $8 \mathrm{Nm}$ to $7.5 \mathrm{Nm}$ to unscrew after 1700 cycles. This indicates that the disc spring design works to retain enough tension in the bolt. The comparison between finite element calculation and experiments showed in the case of gauge 1 order of magnitude in the strain justifying the needs for the experimental results to validate the selected design.

\section{Acknowledgments}

The authors wish to thank the division „Plasma Edge and Wall"'at IPP Garching for its support and in particular Dipl. Ing. (FH) Till Höschen for his assistance with the measurements.

\section{References}

[1] R. Stadler et al.,The in-vessel components of the experiment Wendelstein 7-X, Fusion Engineering and Design, 84 305-308 (2009).

[2] A. Peacock et al., $25^{\text {th }}$ SOFE, San Francisco, California, 2013, to be published.

[3] H. Greuner et al.,High heat flux tests of the Wendelstein 7$\mathrm{X}$ pre-series target elements: Experimental evaluation of the thermo-mechanical behaviour, Fus. Eng. and Design, 82 17131719 (2007).

[4] G. Niemann, Maschinenelemente: Band 1, Springer (2005).

[5] H. Renner et al.,Physical aspects and design of the Wendelstein 7-X divertor, Fusion Science and Technology, 46 318-326 (2004).

[6] M. Ye et al., Thermo-mechanical analysis of the Wendelstein 7-X divertor, Fusion Engineering and Design, 86 1630-1633 (2011).

[7] K. Hoffmann, Eine Einführung in die Technik des Messens mit Dehnungsmeßstreifen, Hottinger Baldwin Messtechnik GmbH, Darmstadt (1987).

[8] X. Peng et al.,Thermo-mechanical analysis of Wendelstein 7-X plasma facing components, Fusion Engineering and Design, 88 1727-1730 (2013).

[9] ANSYS, Inc., ANSYS Workbench 14.5 User's Guide, 2013, https://support.ansys.com/ 